\title{
Pitch discrimination and phase sensitivity in young and elderly subjects and its relationship to frequency selectivity
}

\author{
Brian C.J. Moore \\ Department of Experimental Psychology, University of Cambridge, Downing Street, \\ Cambridge CB2 3EB, England \\ Robert W. Peters \\ Division of Speech and Hearing Sciences, Department of Medical Allied Health Professions, The School \\ of Medicine. The University of North Carolina at Chapel Hill, North Carolina 27599
}

(Received 12 March 1991; accepted for publication 20 January 1992)

\begin{abstract}
Frequency difference limens for pure tones (DLFs) and for complex tones (DLCs) were measured for four groups of subjects: young normal hearing, young hearing impaired, elderly with near-normal hearing, and elderly hearing impaired. The auditory filters of the subjects had been measured in earlier experiments using the notched-noise method, for center frequencies $\left(f_{c}\right)$ of $100,200,400$, and $800 \mathrm{~Hz}$. The DLFs for both impaired groups were higher than for the young normal group at all $f_{c}$ 's $(50-4000 \mathrm{~Hz})$. The DLFs at a given $f_{c}$ were generally only weakly correlated with the sharpness of the auditory filter at that $f_{c}$, and some subjects with broad filters had near-normal DLFs at low frequencies. Some subjects in the elderly normal group had very large DLFs at low frequencies in spite of near-normal auditory filters. These results suggest a partial dissociation of frequency selectivity and frequency discrimination of pure tones. The DLCs for the two impaired groups were higher than those for the young normal group at all fundamental frequencies $\left(f_{0}\right)$ tested $(50,100,200$, and 400 $\mathrm{Hz}$ ); the DLCs for the elderly normal group were intermediate. At $f_{0}=50 \mathrm{~Hz}$, DLCs for a complex tone containing only low harmonics $(1-5)$ were markedly higher than for complex tones containing higher harmonics, for all subject groups, suggesting that pitch was conveyed largely by the higher, unresolved harmonics. For the elderly impaired group, and some subjects in the elderly normal group, DLCs were larger for a complex tone with lower harmonics (1-12) than for tones without lower harmonics (4-12 and 6-12) for $f_{0}$ 's up to 200 $\mathrm{Hz}$. Some elderly normal subjects had markedly larger-than-normal DLCs in spite of nearnormal auditory filters. The DLCs tended to be larger for complexes with components added in alternating sine/cosine phase than for complexes with components added in cosine phase. Phase effects were significant for all groups, but were small for the young normal group. The results are not consistent with place-based models of the pitch perception of complex tones; rather, they suggest that pitch is at least partly determined by temporal mechanisms.
\end{abstract}

PACS numbers: 43.66.Hg, 43.66.Fe, 43.66.Nm, 43.66.Dc [WAY]

\section{INTRODUCTION}

The discrimination of the pitch of complex sounds by the hearing impaired has been studied relatively little, in spite of the fact that it is of both practical and theoretical interest (Hoekstra and Ritsma, 1977; Horst, 1987; Moore and Glasberg, 1987, 1988, 1990a; for a review see Rosen and Fourcin, 1986). One practical interest stems from the relevance of pitch discrimination to speech perception. The pitch patterns of speech indicate which are the most important words in an utterance, they distinguish a question from a statement and they indicate the structure of sentences in terms of phrases. Supplementing speech reading with an auditory signal containing information only about voice pitch can result in a substantial improvement in the ability to understand speech (Risberg, 1974; Rosen et al., 1981; Grant et al., 1985).
The theoretical interest in studying pitch discrimination in impaired listeners stems from the implications of the results for pitch theories. Modern pitch theories differ in the extent to which they assume the use of temporal information, especially temporal information from unresolved high harmonics. In some theories, this information plays little or no role. For example, in the theories of Goldstein (1973) and Terhardt (1974), two stages are assumed to be involved; a complex tone is first analyzed into its sinusoidal components, and then the pitch is derived from the frequencies or pitches of the resolved components (Goldstein, 1973; Terhardt, 1974). In Terhardt's theory, temporal information from unresolved harmonics is assumed to play a secondary role, but is not sufficient on its own to convey pitch; spectral pitch cues are regarded as "essential" (Terhardt, 1977, p. 359 ). We will refer to theories in this category as spectral theories, even though the frequencies of the resolved compo- 
nents may partly be coded in the time patterns of neural impulses.

For a complex tone with equal-amplitude harmonics, only the first five or six harmonics are resolved by normalhearing subjects (Plomp, 1964; Moore et al., 1984). Hence, these theories predict that the pitches of such complex tones should be determined primarily by the first five or six harmonics. This prediction is supported by the finding that the dominant harmonics for pitch tend to be the lower ones (Plomp, 1967; Ritsma, 1967; Moore et al., 1984; Moore et al., 1985). The spectral theories predict that pitch perception should be poor if the lower harmonics are absent, and that the pitch discrimination of complex tones should be unaffected by the relative phases of the components [according to Terhardt's theory, the pitch value perceived may be influenced by relative phase "when sufficient envelope fluctuations are present" (Terhardt, 1977, p. 360), but discrimination should still be determined by the resolved harmonics].

Theories in the second category, called here spectrotemporal theories, assume that the pitch of complex tones is derived from both spectral and temporal information; the spectral analysis performed in the auditory filters is followed by an analysis of the time pattern of the output of each filter, as represented in the patterns of phase locking in the auditory nerve (Moore, 1982, 1989; van Noorden, 1982; Srulovicz and Goldstein, 1983; Moore and Glasberg, 1986a; Patterson, 1987; Meddis and Hewitt, 1991). According to these theories, the pitches of complex tones can be extracted from higher unresolved components as well as from lower resolved components, a prediction supported by experimental results (Ritsma, 1962, 1963; Moore and Rosen, 1979; Houtsma and Smurzynski, 1990). Also, these theories predict that the pitch perception and discrimination of complex tones may be affected by the relative phases of high harmonics, as is also supported by experimental results (Moore, 1977; Houtsma and Smurzynski, 1990).

Subjects with sensorineural hearing loss often have auditory filters that are broader than normal (Pick et al., 1977; Glasberg and Moore, 1986; Tyler, 1986). Broader filters lead to a reduced ability to resolve the harmonics of complex sounds, which, according to the spectral theories, should markedly impair pitch perception and discrimination. The effects of broad filters expected from spectrotemporal theories are somewhat harder to predict. Pitch discrimination might be somewhat impaired by broadened filters, because the temporal information conveying pitch is more ambiguous (Rosen and Fourcin, 1986), but for complex tones with many harmonics, this effect should be small; spectrotemporal theories assume that information can be combined across different frequency regions to resolve ambiguities. However, it is also possible that temporal processing is abnormal in hearing-impaired subjects, in which case pitch discrimination would be markedly impaired. Hypothetically, then, some subjects with broad auditory filters might show reasonably good pitch discrimination, while others (those with abnormal temporal processing) would show marked impairments. The spectrotemporal theories also predict that pitch discrimination by subjects with broader-than-normal auditory filters might be more affected by the relative phases of the components, since even the lower harmonics would interact at the outputs of the auditory filters. There are some data supporting this prediction, although the results tend to be highly variable across subjects (Rosen and Fourcin, 1986; Moore and Glasberg, 1987, 1988, 1990a).

Consider now the pitch discrimination of pure tones. One type of spectral theory suggests that the pitch discrimination of pure tones is determined entirely by place mechanisms. According to such a theory, normal auditory filters should be associated with normal pitch discrimination, whereas broadened auditory filters should lead to poor pitch discrimination. On the other hand, the pitch of pure tones could be determined primarily by temporal mechanisms, for example by the measurement of neural interspike intervals (Goldstein and Srulovicz, 1977). In this case, some subjects with broad auditory filters might show normal pitch discrimination for pure tones, but an abnormality in temporal processing could lead to poor pitch discrimination even if the auditory filters were normal. Between these two extremes, several workers have suggested that the pitch discrimination of pure tones might involve both place and time information (e.g., Srulovicz and Goldstein, 1983). In this case, either broad auditory filters or poor temporal processing could lead to poor pitch discrimination.

In the experiments reported here, we measured pitch discrimination in normal and hearing-impaired subjects for both pure and complex tones. The complex tones were chosen to contain either low harmonics (1-5) which would be primarily resolvable at fundamental frequencies above 100 $\mathrm{Hz}$, high harmonics (6-12) that would be largely unresolvable, or both resolvable and unresolvable harmonics (1-12). In addition, a complex tone was used that contained harmonics 4-12; the lower few harmonics in this tone would be resolvable by a normal ear, but would probably not be resolvable by an ear with reduced frequency selectivity. The complex tones had components added either in cosine phase, or in alternating sine-cosine phase.

Four groups of subjects were tested: young subjects with normal hearing; young subjects with impaired hearing; elderly subjects with normal or near-normal hearing; and elderly subjects with impaired hearing. "Hearing" in this context refers to absolute thresholds. We had previously measured auditory filter shapes in these subjects over the range $100-800 \mathrm{~Hz}$ (Moore et al., 1990; Peters and Moore, $1992 a, b)$. These filter shapes were measured using the notched-noise method (Patterson, 1976; Patterson and Moore, 1986), and a summary of the results will be presented later.

The comparison of the first and third groups, and the second and fourth groups, was intended to allow us to determine whether age per se has an effect on pitch discrimination. The first group should have both normal frequency selectivity and normal temporal processing. The second and fourth groups contained subjects who mostly had abnormal frequency selectivity for at least some center frequencies (Peters and Moore, 1992a). We expected that some of these subjects might also have impaired temporal processing. Finally, the third group contained some subjects with almost 
normal auditory filtering (Peters and Moore, 1992b), but who might have impaired temporal processing; it has often been suggested that aging is associated with impaired temporal processing (Humes and Christopherson, 1991).

\section{METHOD}

\section{A. Subjects}

Group one was composed of five young, normal-hearing subjects. Their thresholds were close to $0 \mathrm{~dB} H \mathrm{HL}$ at all audiometric frequencies. Group two was composed of five subjects aged 24 to 34 with hearing impairments of cochlear origin. Group three contained 10 subjects, aged 62 to 83, with normal or near-normal audiograms at $2000 \mathrm{~Hz}$ and below. Although most subjects in this group had thresholds somewhat above $0 \mathrm{~dB} \mathrm{HL}$ at some frequencies, thresholds for the ear tested did not exceed $25 \mathrm{~dB} \mathrm{HL}$ for frequencies of $2000 \mathrm{~Hz}$ and below. Group four contained seven hearing-impaired subjects, aged 66 to 82 . The audiograms for the test ears of all subjects in groups two, three and four are shown in Fig. 1. The elderly subjects were all alert, readily able to follow instructions, and with no obvious memory or concentration problems. Most were retired professional persons, still leading active lives.

Subjects in groups two and four had been previously examined in an audiology clinic and diagnosed as having sensorineural loss. They were also examined by an otologist to insure that the ear canal was not obstructed or liable to collapse and to exclude other obvious otopathological conditions not related to normal processes of aging or of hearing loss. The audiograms of groups two and four were matched as closely as possible, both in mean values and in the range of thresholds, to insure that any effects of age would not be confounded by absolute threshold differences between the two groups. The match was generally good for frequencies up to $2000 \mathrm{~Hz}$ (see Fig. 1).

Table I gives a summary of the characteristics of the four subject groups, including the parameters of the auditory filters derived in our earlier studies of the same subjects (Moore et al., 1990; Peters and Moore, 1992a,b). The filters were derived using the notched-noise method (Patterson, 1976), with an overall noise level of either $77 \mathrm{~dB}$ SPL or 87 dB SPL; the higher level was used for hearing-impaired subjects with relatively large losses, to insure that masked thresholds were above absolute threshold even at large notch widths. The auditory filters were assumed to have the form of the roex $(p, r)$ filter described by Patterson et al. (1982):

$$
W(g)=(1-r)(1+p g) \exp (-p g)+r,
$$

where $g$ is the normalized deviation from the center of the filter (deviation from center frequency divided by center frequency), $p$ is a parameter determining the shape of the passband of the filter, and $r$ is a parameter that places a dynamic range limitation on the filter. The value of $p$ was allowed to differ for the upper and lower halves of the filter. The upper and lower $p$ values are called $p_{u}$ and $p_{l}$, respectively. The value of $r$ was assumed to be the same for the two sides of the filter. Table I gives the means and standard deviations of the parameters $p_{u}, p_{t}$ and $r$ for each group. It also gives the equivalent rectangular bandwidths (ERBs) of the filters, ex-

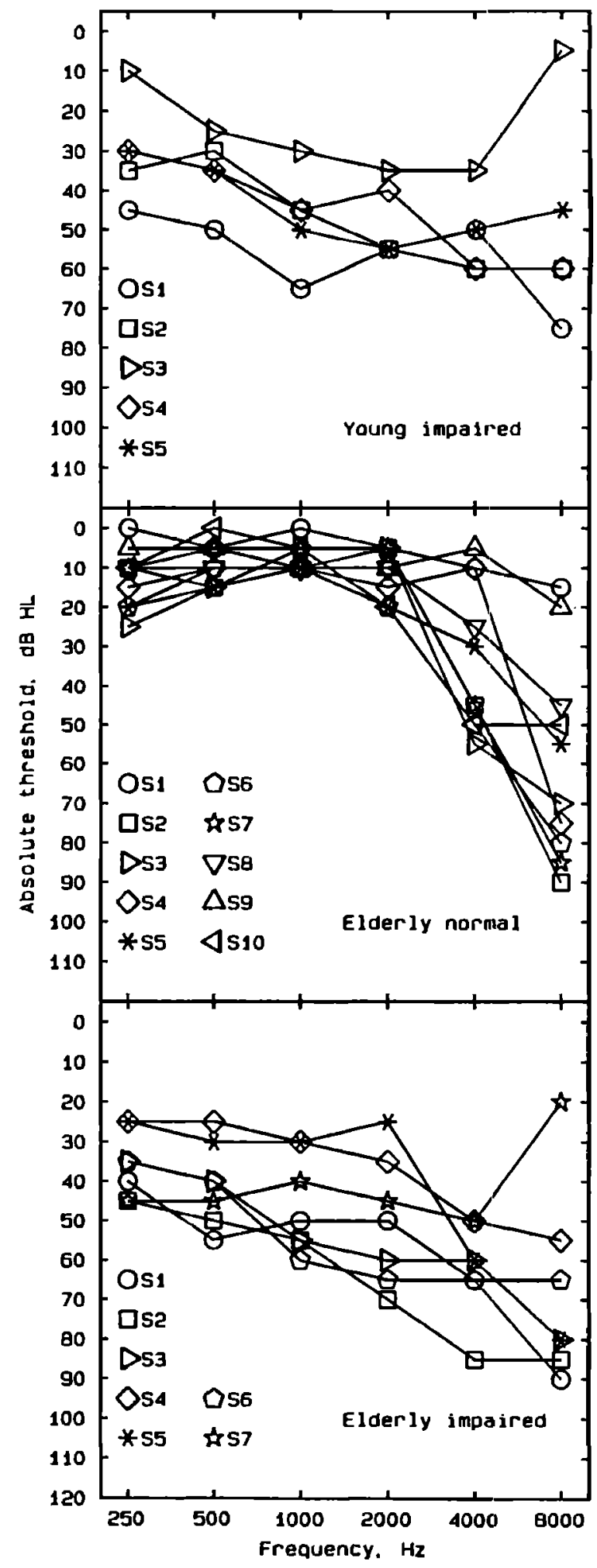

FIG. 1. Absolute thresholds in $\mathrm{dB} \mathrm{HL}$ for the young hearing-impaired subjects (top panel), the elderly subjects with near-normal hearing at low frequencies (middle panel), and the elderly subjects with impaired heanng (bottom panel). These thresholds were obtained by conventional audiometry.

pressed as a proportion of center frequency. The values of the ERBs for individual subjects in groups two, three and four are shown in Fig. 2. The subjects in the elderly normal group had close to normal auditory filters al center frequen- 
TABLE I. Characteristics of the four groups of subjects showing means and standard deviations (in parentheses) of the absolute thresholds (dB SPL) at each test frequency, $f_{c}(\mathrm{~Hz})$, and the parameters of the auditory filters, as derived by Moore et al. (1990) and Peters and Moore (1992a,b). The absolute thresholds were obtained using a three-altemative forced-choice task tracking the $79.4 \%$ correct point on the psychometric function.

\begin{tabular}{|c|c|c|c|c|c|c|}
\hline Group & $f_{c}$ & $\begin{array}{l}\text { Absolute } \\
\text { threshold }\end{array}$ & $p_{l}$ & $P_{u}$ & $r$ & ERB \\
\hline \multirow[t]{4}{*}{ Young normal } & 100 & $33(3)$ & $9.1(3.0)$ & $14.2(3.0)$ & $-54(19)$ & $0.39(0.10)$ \\
\hline & 200 & $20(4)$ & $14.5(1.2)$ & $21.3(3.0)$ & $-63(17)$ & $0.23(0.02)$ \\
\hline & 400 & $16(3)$ & $15.2(1.4)$ & $23.5(2.9)$ & $-60(13)$ & $0.22(0.02)$ \\
\hline & 800 & $9(4)$ & $18.1(3.3)$ & $23.6(2.8)$ & $-55(18)$ & $0.20(0.02)$ \\
\hline \multirow[t]{4}{*}{ Young impaired } & 100 & $48(14)$ & $8.1(4.1)$ & $12.5(3.7)$ & $-39(19)$ & $0.52(0.36)$ \\
\hline & 200 & $48(14)$ & $12.1(4.8)$ & $16.0(5.4)$ & $-34(13)$ & $0.34(0.11)$ \\
\hline & 400 & $42(13)$ & $10.1(3.9)$ & $18.6(10.9)$ & $-30(7)$ & $0.36(0.15)$ \\
\hline & 800 & $46(15)$ & $14.1(3.7)$ & $13.2(5.0)$ & $-28(10)$ & $0.36(0.14)$ \\
\hline \multirow[t]{4}{*}{ Elderly normal } & 100 & $47(11)$ & $5.8(3.7)$ & $9.9(3.3)$ & $-49(19)$ & $0.66(0.28)$ \\
\hline & 200 & $31(13)$ & $11.8(2.1)$ & $16.4(4.4)$ & $-51(8)$ & $0.31(0.07)$ \\
\hline & 400 & $19(7)$ & $15.4(1.6)$ & $20.6(3.8)$ & $-55(13)$ & $0.23(0.02)$ \\
\hline & 800 & $16(7)$ & $21.0(3.9)$ & $23.3(2.2)$ & $-52(3)$ & $0.19(0.02)$ \\
\hline \multirow[t]{4}{*}{ Elderly impaired } & 100 & $56(9)$ & $8.7(4.8)$ & $9.0(3.6)$ & $-25(14)$ & $0.56(0.26)$ \\
\hline & 200 & $48(11)$ & $7.1(6.3)$ & $11.1(7.5)$ & $-34(16)$ & $0.76(0.59)$ \\
\hline & 400 & $48(9)$ & $10.7(4.3)$ & $15.7(10.8)$ & $-32(11)$ & $0.45(0.29)$ \\
\hline & 800 & $45(10)$ & $14.4(4.7)$ & $19.0(7.2)$ & $-25(9)$ & $0.30(0.12)$ \\
\hline
\end{tabular}

cies of 200,400 , and $800 \mathrm{~Hz}$. At $100 \mathrm{~Hz}$, there was considerable individual variability, with some subjects showing normal filters and some showing broad filters.

\section{B. Stimuli}

All stimuli were digitally generated using a 12-bit digital-to-analog converter (DAC). The sampling rate $f_{s}$ was 5 $\mathrm{kHz}$ for fundamental frequencies $\left(f_{0}\right)$ of 50 and $100 \mathrm{~Hz}$ and for pure tones up to $100 \mathrm{~Hz}$, and was $10 \mathrm{kHz}$ for $f_{0}=200$ and $400 \mathrm{~Hz}$ and for pure tones above $100 \mathrm{~Hz}$. The output of the DAC was low-pass filtered at $0.44 f_{s}$, using two Krohn-Hite filters (model 3550, slope $48 \mathrm{~dB} /$ oct). All signals had 10-ms raised-cosine ramps and a steady-state portion of $200 \mathrm{~ms}$. The interval between successive stimuli in a trial was $300 \mathrm{~ms}$. The level of the stimuli was determined by a Wilsonics programmable attenuator (PATT) and a manual attenuator preceding the Sennheiser HD424 earphone.

\section{Complex tones}

The complex tones were harmonic complexes composed of equal-amplitude harmonics with fundamental frequencies $\left(f_{0}\right)$ of $50,100,200$, and $400 \mathrm{~Hz}$ (the range of voice pitch). Each component had a level of $75 \mathrm{~dB}$ SPL, chosen to be above threshold for all subjects. It was checked that the stimuli were comfortably loud for each subject. The tones contained harmonics $1-12,6-12,4-12$, and 1-5. For $f_{0}=400$ $\mathrm{Hz}$, the highest harmonic number was 10 (giving complexes with harmonics 1-10, 6-10,4-10, and 1-5) to insure that all harmonics were audible for all subjects; absolute thresholds were typically increasingly elevated above $4000 \mathrm{~Hz}$ for most of the hearing-impaired subjects. For simplicity, however, when referring to harmonic content, 1-12, 6-12, 4-12, and $1-5$ will be used throughout the paper.

The components of the harmonic complexes were added in one of two phase relationships, all cosine phase or alternating cosine and sine phase. The former results in a wave- form with prominent peaks and low amplitudes between the peaks. The latter results in a waveform with a much flatter envelope.

\section{Pure tones}

Frequency discrimination of pure tones was measured for frequencies of $50,100,200,400,800,1000,1200,2000$, and $4000 \mathrm{~Hz}$. The level of the tones was $25 \mathrm{~dB}$ above the absolute threshold at the test frequency ( $25 \mathrm{~dB} \mathrm{SL}$ ).

\section{Procedure}

DLs were measured using an adaptive three-interval, three-alternative forced-choice method. Each trial consisted of three observation intervals, marked by lights. In two of the intervals, the frequency ( $\operatorname{or} f_{0}$ ) of the stimulus was the same, while in the third, selected at random, the frequency (or $f_{0}$ ) was higher. The task of the subject was to select the observation interval containing the higher frequency. Feedback was provided by lights on the response box. A three-down one-up rule was used to estimate the frequency difference corresponding to the $79.4 \%$ correct point on the psychometric function. The difference in frequency between the signal and no-signal intervals started at $10 \%$ of the center frequency (or of $f_{0}$ ). For subjects and/or conditions where thresholds were close to $10 \%$, the starting difference was increased to $20 \%$. After three consecutive correct responses, the difference in frequency was decreased by a factor of 1.4 , while after one incorrect response, the difference was increased by the same factor. Twelve turnpoints were obtained and threshold was taken as the geometric mean of the frequency differences at the last eight turnpoints. For a few subjects, only eight turnpoints were obtained, and threshold was estimated from the last four. At least three threshold estimates were obtained for each condition. Subjects were tested individually in a single-walled sound-attenuating chamber. 


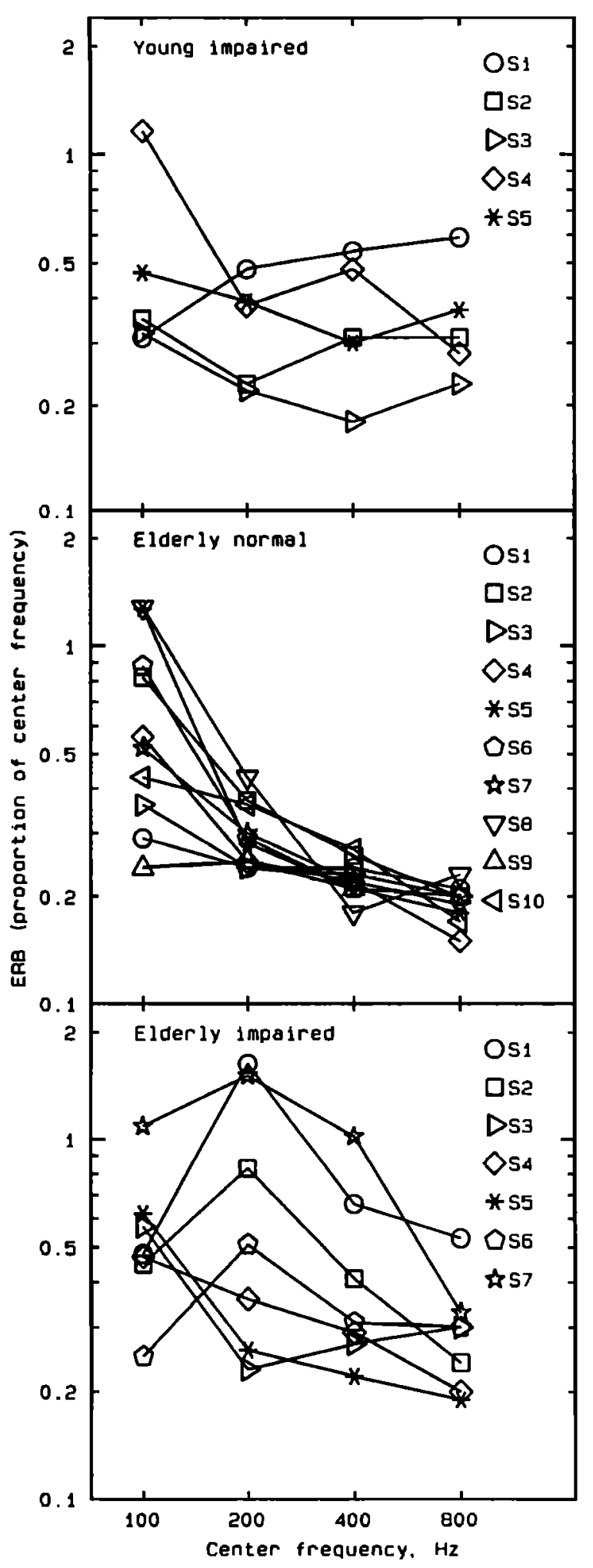

FIG. 2. Equivalent rectangular bandwidths (ERBs) of the auditory filters for the young hearing-impaired subjects (top panel), the elderly subjects with near-normal hearing at low frequencies (middle panel), and the elderly subjects with impaired hearing (bottom panel).

Subjects were given only a small amount of training before data collection began. However, the three-interval task used by us appears to be easier to learn than the two-interval task more commonly used, and there was no evidence of subjects improving during the course of the experiment. It seems likely that subjects find it easier to pick the "odd one out" than to decide whether a pitch went up or down.

\section{RESULTS}

\section{A. Frequency discrimination of sinusoids}

The geometric mean thresholds for the frequency discrimination of sinusoids (DLFs) are plotted separately for each group in Fig. 3. DLFs are expressed as a percentage of frequency and plotted on a logarithmic scale. Error bars indicate standard deviations (s.d.) across subjects. The DLFs for the young normal subjects are broadly in line with previous work (Moore, 1973; Wier et al., 1977; Nelson et al., 1983), except that overall they are somewhat higher than typically reported in the literature. The ratios of our mean DLFs to those derived from the equation suggested by Nelson et al. $[\log (\mathrm{DLF})=a \sqrt{f}+k-m / \mathrm{SL}$, where $f$ is frequency, SL is sensation level, and the constants have values: $a=0.023, k=-0.25$, and $m=4.3]$ are 2.2, 2.0, 2.6, 1.8, $1.4,1.7,2.1,2.0$, and 2.1 , for center frequencies of 50,100 , $200,400,800,1000,1200,2000$, and $4000 \mathrm{~Hz}$, respectively. The fact that our thresholds are about a factor of two larger than predicted by Nelson et al. can be attributed mainly to the fact that our three-interval forced-choice procedure tracking $79.4 \%$ correct (corresponding to $d^{\prime}=1.6$ ) gives thresholds about twice as high as the two-interval forcedchoice task tracking $70.7 \%$ correct (corresponding to $d^{\prime}=0.78$ ) typically used in earlier studies.

Both groups of impaired subjects showed larger DLFs than normal, a result consistent with previous studies (Zurek and Formby, 1981; Tyler et al., 1983; Moore and Glasberg, 1986b). However, individual differences were quite large. The elderly normal group also had larger than normal DLFs, similar to those of the elderly-impaired group, except at 2 and $4 \mathrm{kHz}$, where their DLFs were somewhat smaller. The large DLFs in the elderly normal group occurred even in the range $200-800 \mathrm{~Hz}$, where both their absolute thresholds and their auditory filters were almost normal. This suggests a partial dissociation between frequency selectivity and frequency discrimination. This dissociation is illustrated more clearly in Fig. 4, which shows individual results for three subjects from the elderly normal group, selected because their auditory filters were very close to normal at all center frequencies tested $(100-800 \mathrm{~Hz})$. Error bars for the individual elderly subjects indicate the s.d. of the three estimates for each condition. Error bars for the young normal group (top-left panel) show s.d.'s calculated in the same way for each subject and then averaged across subjects.

One elderly subject, S9, shows near-normal DLFs at most center frequencies, except, perhaps, at $100 \mathrm{~Hz}$. The other subjects show markedly elevated DLFs at all center frequencies. Their DLFs are clearly higher than normal in the range $100-800 \mathrm{~Hz}$ where their auditory filters are normal. This is not consistent with a strict place model of frequency discrimination. It is, however, consistent with the use of temporal information in frequency discrimination; subjects $\$ 3$ and $\$ 4$ may have impaired temporal processing 


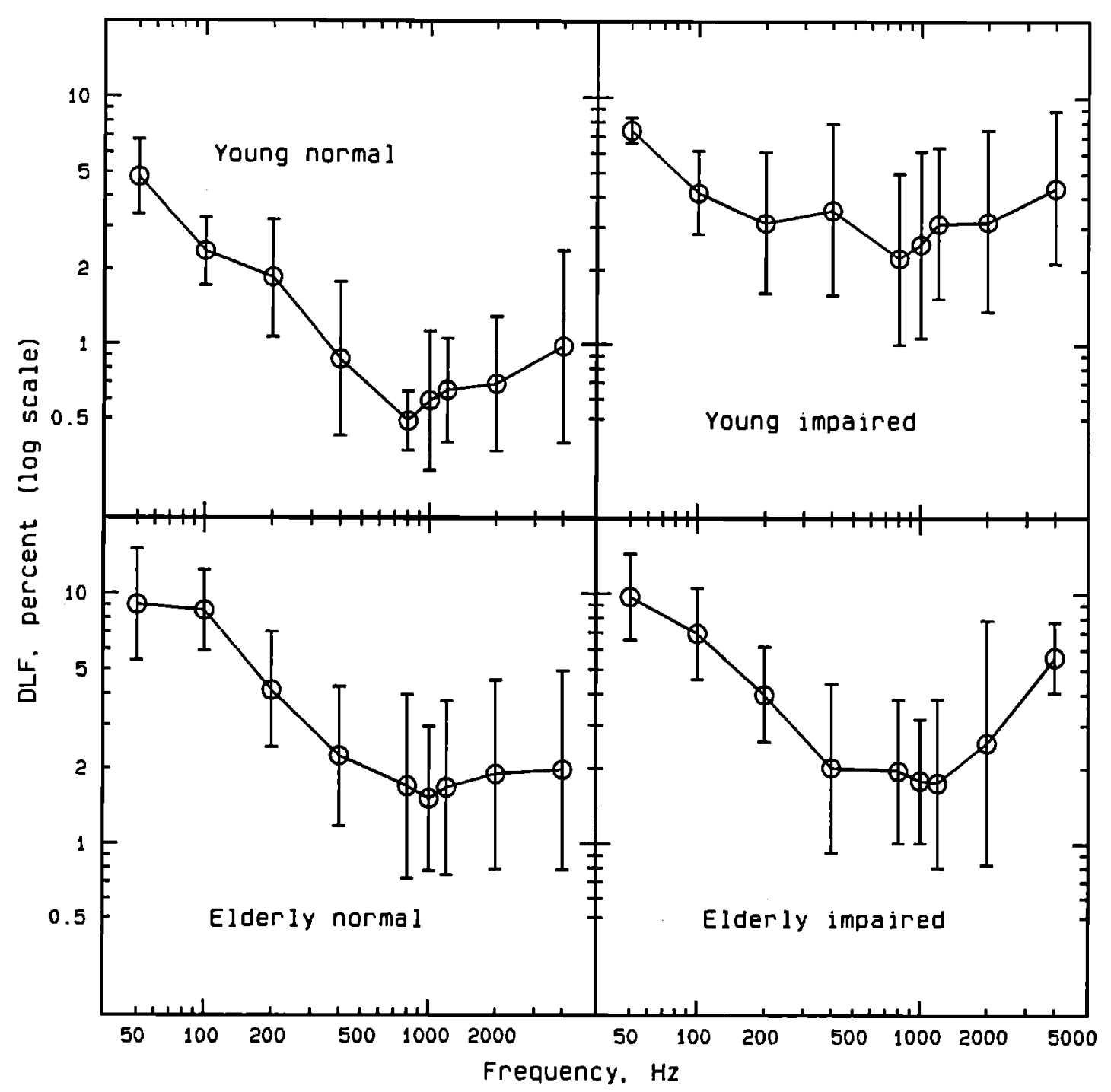

FIG. 3. Frequency DLs for sinusoids (DLFs), shown separately for each group of subjects. The DLFs were calculated as geometric means, and are plotted as a percentage of center frequency $\left(f_{c}\right)$, on a logarithmic scale. Error bars show standard deviations across subjects.

in spite of their normal auditory filters.

Another example of a dissociation between frequency selectivity and frequency discrimination comes from consideration of the individual results for the impaired subjects. Figure 5 shows results for two elderly impaired subjects (S1 and S4), one young impaired subject (S2) and the mean results for young normal subjects. Elderly subject $\mathbf{S 1}$ had broader-than-normal auditory filters at each center frequency tested; the ERBs, expressed as a proportion of center frequency $\left(f_{c}\right)$, were $0.48,1.54,0.66$, and 0.50 , for $f_{c}=100$, 200,400 , and $800 \mathrm{~Hz}$, respectively. This subject had DLFs that were only slightly higher than normal at low frequencies. Elderly subject $\mathbf{S} 4$ and young subject $\mathbf{S} 2$ both had nearnormal auditory filters from $f_{c}=100$ to $800 \mathrm{~Hz}$. Both had DLFs within the normal range for frequencies from 400 to $2000 \mathrm{~Hz}$. However, elderly subject $\mathbf{4} 4$ had larger-than-normal DLFs at low frequencies, particularly at 100 and 200 $\mathrm{Hz}$. Indeed, for frequencies of 50,100 , and $200 \mathrm{~Hz}$, elderly subject S4 had larger DLFs than elderly subject S1, in spite of the fact that $S 1$ had broad low-frequency auditory filters and $\mathrm{S} 4$ had near-normal filters. This relatively poor performance by S4 is unlikely to be explicable in terms of a generally poor ability to perform frequency discrimination, since at midrange center frequencies $\mathbf{S 4}$ had smaller DLFs than $\mathbf{S 1}$.

The partial dissociation of frequency selectivity and frequency discrimination is confirmed by an analysis of the Pearson's product-moment correlations between the DLFs and the measures of frequency selectivity. There was generally only a small correlation between the ERB at a given center frequency and the DLF at that same frequency. For the combined data of the two hearing-impaired groups (2 and 4 ), the correlations were $0.23,0.17,0.68$, and -0.01 for $f_{c}=100,200,400$, and $800 \mathrm{~Hz}$, respectively. After partialing out the effect of absolute threshold at each frequency (Kendall and Stuart, 1967), these correlations became $-0.08,0.05,0.45$, and -0.07 , none of which is significant. 


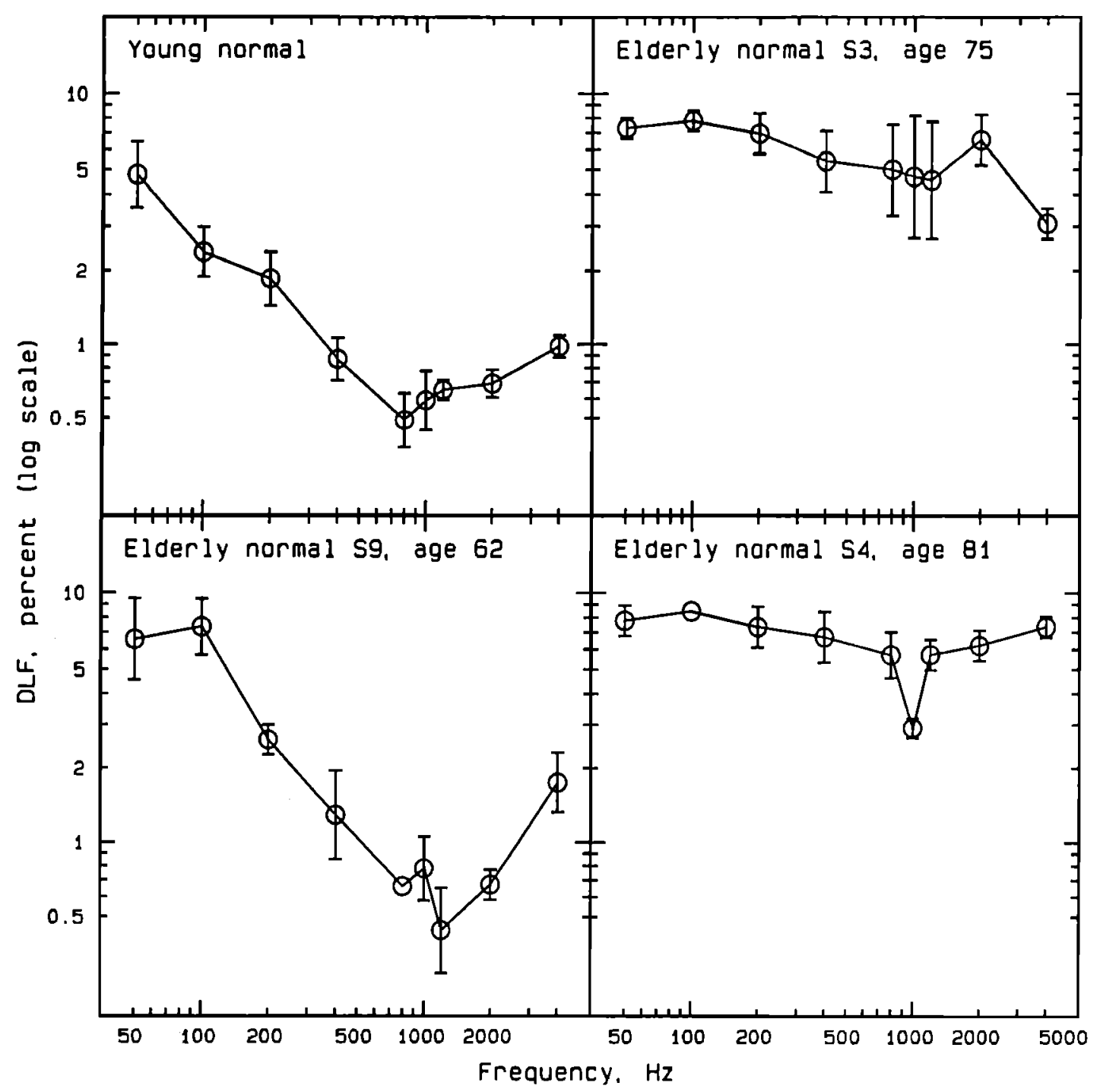

FIG. 4. DLFs for the young normal group and for three individual subjects from the elderly normal group, chosen because their auditory filters were almost normal at low center frequencies. Error bars for individual subjects show sample standard deviations, calculated from the three threshold estimates for each $f_{c}$. Error bars for the young normal group show sample standard deviations calculated in the same way and then averaged across subjects.

Similarly low correlations were found between the DLFs and the values of $p_{4}$ and $p_{l}$ at each center frequency. These results are similar to those reported by Tyler et al. (1983) and by Glasberg and Moore (1989). The DLFs generally showed small positive correlations with audiometric thresholds (as shown in Fig. 1). The correlations were generally higher between the DLFs and absolute thresholds at the same frequency (e.g., 1,2 , or $4 \mathrm{kHz}$, where the correlations were $0.35,0.54$, and 0.18 , respectively) than between the DLFs and absolute thresholds at either higher or lower frequencies. This suggests that information about the frequency of each tone was being extracted primarily from the frequency region (place) corresponding to that tone, rather than from remote regions.

It should be noted that the low correlations between the DLFs and the measures of frequency selectivity and absolute thresholds do not result simply from poor reliability in the measures of the DL.Fs. This is indicated by the fact that DLFs at adjacent frequencies were quite highly correlated. For example, for the combined results of the two hearingimpaired groups, the DLFs at 200 and $400 \mathrm{~Hz}$ showed a correlation of 0.73 , those at 400 and $800 \mathrm{~Hz}$ showed a correlation of 0.93 , those at 800 and $1000 \mathrm{~Hz}$ showed a correlation of 0.64 , and those at 1000 and $1200 \mathrm{~Hz}$ showed a correlation of 0.77 .

In summary, both the elderly and young hearing-impaired groups showed impaired frequency discrimination, and some subjects in the elderly normal group also showed impaired frequency discrimination. A partial dissociation of frequency selectivity and frequency discrimination is indicated by three aspects of the results: (1) some subjects had normal auditory filters at low center frequencies but had abnormally large DLFs; (2) one subject had broader-thannormal auditory filters at low center frequencies but had 
almost normal DLFs; (3) The correlations between the DLFs and measures of frequency selectivity were small, and after partialing out the effect of absolute threshold they were not significant. Overall, the results do not support the idea of a purely place-based mechanism of frequency discrimination for pure tones at low center frequencies.

\section{B. Frequency discrimination of complex tones}

The geometric mean thresholds for the frequency discrimination of complex tones (DLCs) are plotted separately for each group in Fig. 6. DLCs are expressed as a percentage of $f_{0}$ and plotted on a logarithmic scale. Each symbol represents results for a particular harmonic complex, as indicated by the key in the upper right panel. Standard deviations across subjects are not shown, to avoid clutter in the figure, but the s.d. were comparable to those found for pure tones (see Figs. 3, 4, and 5). The results have been averaged across the two phase conditions; phase effects will be discussed later.

The DLCs for the normal subjects are 2-2.5 times larger than reported in the literature (e.g., Moore et al., 1984; Moore and Glasberg, 1990b). As for the DLFs, most of the discrepancy can be explained by the higher value of $d^{\prime}$ tracked in our experiments. However, our subjects were not highly trained, and this, or individual differences, may account for the small remaining discrepancy (a factor less than 1.3).

As was found for the DLFs, performance is clearly worse for the two hearing-impaired groups than for the young normal group. DLCs for the elderly normal group are also higher than for the young normal group, especially at low $f_{0}$ 's. Indeed, at $f_{0}=50 \mathrm{~Hz}$, DLCs for the elderly normal group are similar to those for the two impaired groups. For all four groups, DLCs for $f_{0}=50 \mathrm{~Hz}$ are higher for the complex containing harmonics 1-5 than for any of the other

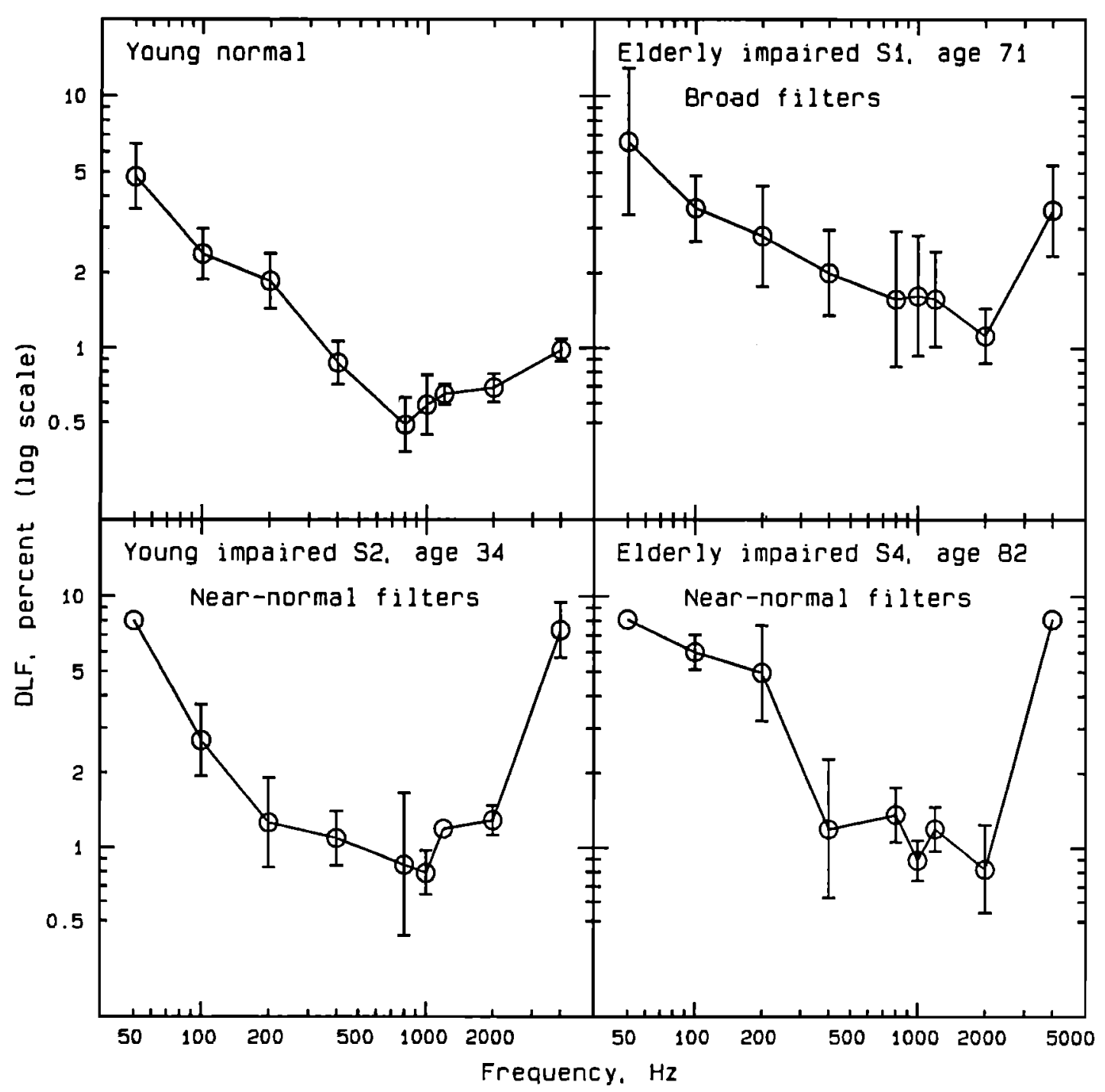

FIG. 5. DLFs for the young normal group and for three individual subjects. Elderly impaired subject $\$ 1$ had broad auditory filters at low center frequencies. Young impaired subject S2 and elderly impaired subject $\mathbf{4}$ had near-normal auditory filters at low center frequencies. 


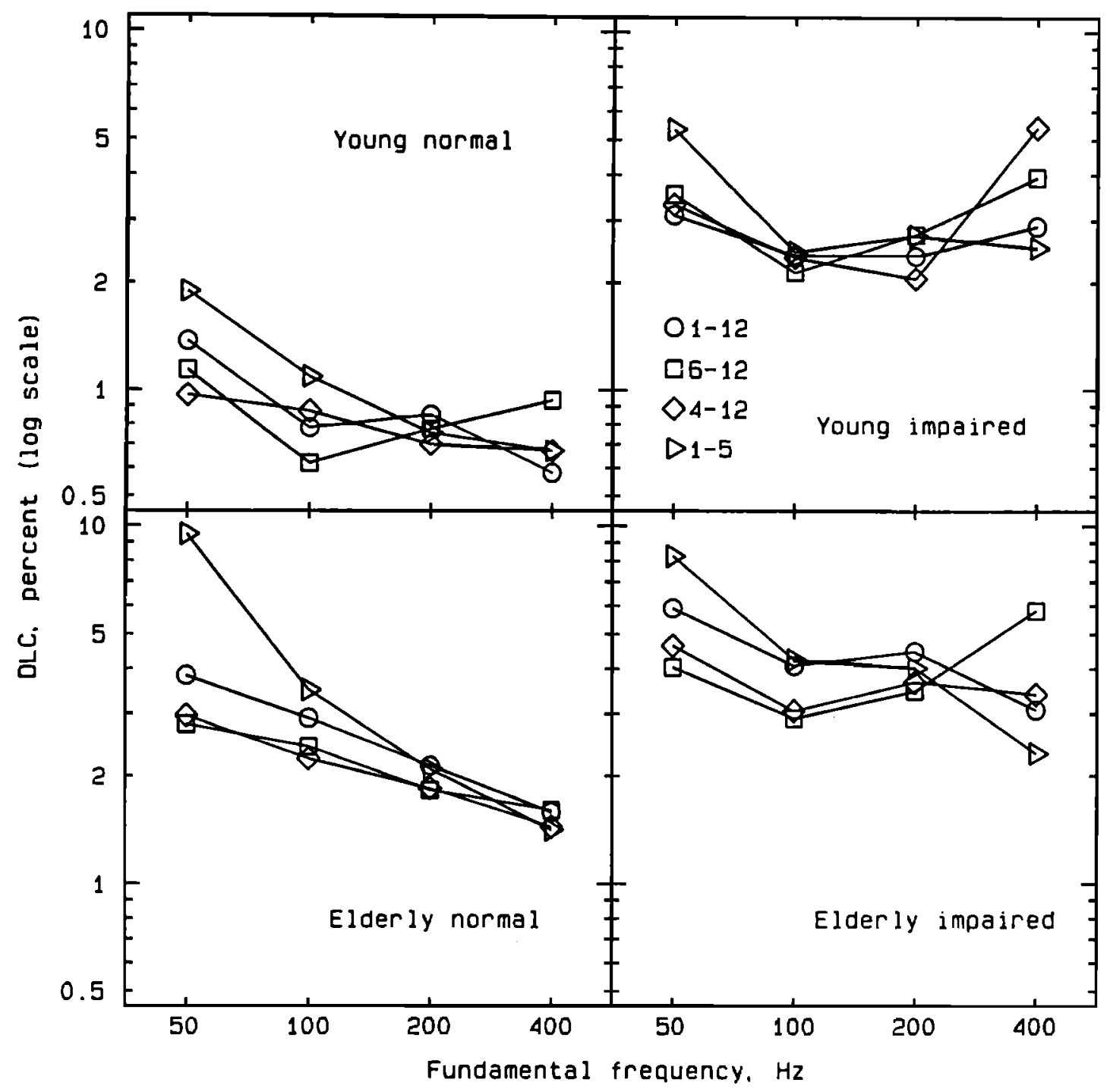

FIG. 6. Frequency DLs for complex tones (DLCs), for each group of subjects. The DLCs were calculated as geometric means, and are plotted as a percentage of $f_{0}$, on a logarithmic scale. Each symbol represents a different harmonic complex. Results have been averaged for complex tones with components added in cosine phase and alternating phase.

complexes. This suggests that, at very low $f_{0}$ 's, pitch is extracted primarily from harmonics above the fifth. This is consistent with results presented by Moore and Glasberg (1988, 1990a). In contrast, at $f_{0}=400 \mathrm{~Hz}$, the complexes containing only high harmonics (6-12 and 4-12) tend to be the most poorly discriminated, especially by the two impaired groups. These results indicate that the dominant region for pitch is not fixed in harmonic number, but shifts upward in harmonic number as $f_{0}$ decreases, as suggested by earlier work (Plomp, 1967; Patterson and Wightman, 1976).

The results for each group were subjected to an analysis of variance (ANOVA) with factors: subject, harmonic content, phase (cosine or alternating), and $f_{0}$. The variance associated with the four-way interaction was used as an estimate of the residual variance. The analysis program used (GENSTAT) gave estimates of the standard errors of the differences between pairs of mean scores (e.g., between com- plexes with different harmonic contents). These standard errors were used to assess the significance of the differences between means (Alvey et al., 1982, p. 81). The results were as follows.

For the young normal group, all of the main factors were significant: for subject, $F(4,36)=178.7, p<0.001$; for harmonic content, $F(3,36)=15.8, p<0.001$; for phase, $F(1,36)=5.7, p=0.022$; for $f_{0}, F(3,36)=79.3, p<0.001$. The DLCs for alternating phase were larger than those for cosine phase; more detail will be presented later. There was a significant interaction of harmonic content and $f_{0}$ $[F(9,36)=12.7, p<0.001]$; at $f_{0}=50 \mathrm{~Hz}$, DLCs were higher for complex 1-5 than for the other complexes, while at $f_{0}=400 \mathrm{~Hz}$, DLCs were higher for complex 6-12 than for the other complexes. The fact that DLCs at $f_{0}=50 \mathrm{~Hz}$ were significantly larger for complex 1-5 than for the other complexes, is contrary to what might have been expected from spectral theories of pitch perception. The interactions of 
phase with harmonic content and with $f_{0}$ were not significant.

For the young impaired group, the main effect of harmonic content was not significant, but the other main effects were: for subject, $F(4,33)=57.2, p<0.001$; for phase, $F(1,33)=9.9, p=0.004$; for $f_{0}, F(3,33)=10.0, p<0.001$. The DLCs for alternating phase were larger than those for cosine phase. There was a significant interaction of harmonic content and $f_{0}[F(9,33)=3.5, p=0.004]$; at $f_{0}=50 \mathrm{~Hz}$, DLCs were higher for complex 1-5 than for the other complexes, while at $f_{0}=400 \mathrm{~Hz}$, DLCs were higher for complexes 4-12 and 6-12 than for the other complexes. The interactions of phase with harmonic content and with $f_{0}$ were not significant.

For the elderly normal group, all of the main factors were significant: for subject, $F(9,75)=57.9, p<0.001$; for harmonic content, $F(3,75)=71.0, p<0.001$; for phase, $F(1,75)=8.9, \quad p=0.004 ; \quad$ for $f_{0}, \quad F(3,75)=168.4$, $p<0.001$. DLCs were significantly larger for complex $1-5$ than for the other complexes $(p<0.01)$, although this occurred only at $f_{0}=50$ and $100 \mathrm{~Hz}$. Also, DLCs for complex 1-12 were larger than DLCs for complexes 6-12 and 4-12 (both $p<0.01$ ). This indicates that adding lower harmonics to a complex tone can impair pitch discrimination. DLCs for alternating phase were larger than those for cosine phase. There was a significant interaction of harmonic content and $f_{0}[F(9,75)=46.7, p<0.001]$; at $f_{0}=50 \mathrm{~Hz}$, DLCs were higher for complex 1-5 than for the other complexes, while at other $f_{0}$ 's DLCs were similar for all complexes. The interaction of phase with harmonic content was not significant, but the interaction of phase with $f_{0}$ was significant $[F(3,75)=11.9, p<0.001]$; phase effects were greatest at the lowest $f_{0}$.

For the elderly impaired group, all of the main factors were significant: for subject, $F(7,54)=270.0, p<0.001$; for harmonic content, $F(3,54)=15.4, p<0.001$; for phase, $F(1,54)=10.5, \quad p=0.002$; for $f_{0}, \quad F(3,54)=82.5$, $p<0.001$. DLCs were significantly larger for complex 1-5 than for the other complexes $(p<0.05)$. Also DLCs for complex 1-12 were larger than those for complex 6-12 $(p<0.05)$ and $4-12(p<0.001)$. These effects occurred at $f_{0}=50$ and $100 \mathrm{~Hz}$. As for the elderly normal group, this indicates that adding lower harmonics to a complex tone can impair pitch discrimination. DLCs for alternating phase were larger than those for cosine phase. There was a significant interaction of harmonic content and $f_{0}$ $[F(9,54)=37.9, p<0.001]$; at $f_{0}=50 \mathrm{~Hz}$, DLCs were higher for complex 1-5 than for the other complexes, while at $f_{0}=400 \mathrm{~Hz}$ DLCs were higher for complexes 6-12 and 4 12 than for the other complexes. The interactions of phase with harmonic content and with $f_{0}$ were not significant.

The pattern of these results lends support to spectrotemporal theories of pitch perception. It is clear that, at low $f_{0}$ 's, pitch is derived primarily from the higher, unresolved harmonics, both for normal and impaired subjects. For the two elderly groups, performance was worse for complex 1-12 than for complexes $4-12$ or $6-12$, indicating that adding lower harmonics to a complex tone can actually impair performance. This may happen because, when auditory filters are broader than normal, adding lower harmonics can create more complex waveforms at the outputs of the auditory filters, making temporal analysis more difficult. It should be noted that several of the subjects in the elderly normal group did have broader-than-normal auditory filters at center frequencies of 100 and $200 \mathrm{~Hz}$ (see Fig. 2).

Figure 7 shows DLCs for the same subjects whose DLFs were shown in Fig. 4. Recall that these subjects were selected from the elderly normal group because their auditory filters were essentially normal over the range $100-800 \mathrm{~Hz}$. For these subjects, DLCs are generally similar for the complexes $1-12,6-12$, and $4-12$. Thus the presence or absence of the lower harmonics has little effect on performance when auditory filtering is normal. Parallel to what was found for the DLFs, the DLCs for elderly normal subjects S3 and S4 are markedly worse than normal, in spite of their near-normal auditory filters. In contrast, elderly normal subject $\$ 9$ shows DLCs within the range found for young normal subjects. Thus subjects with normal auditory filters can have quite different abilities to discriminate the pitches of complex tones.

The DLCs were mostly only weakly correlated with the measures of frequency selectivity. Considering the results for the two hearing-impaired groups together, the correlation of the DLCs with the ERBs was typically about 0.4 . However, the DLC for $f_{0}=200 \mathrm{~Hz}$ was correlated with the ERB for $f_{c}=200 \mathrm{~Hz}(r=0.88, p<0.01)$ and $f_{c}=400 \mathrm{~Hz}$ $(r=0.79, p<0.01)$ and these correlations remained high ( $r=0.90$ and 0.70 ) after partialing out the effect of the absolute threshold at 200 and $400 \mathrm{~Hz}$, respectively. Also, the DLC for $f_{0}=100 \mathrm{~Hz}$ was correlated with the ERB for $f_{c}=400 \mathrm{~Hz}(r=0.77, p<0.01)$, and the correlation remained reasonably high $(r=0.61)$ after partialing out the effect of absolute threshold at $400 \mathrm{~Hz}$. The DLCs for $f_{0}=50$ and $400 \mathrm{~Hz}$ were not significantly correlated with any of the measures of frequency selectivity. Thus, while there is a trend for large DLCs to be associated with poor frequency selectivity, the relationship does not seem to be a close one.

The DLCs showed moderate positive correlations with the absolute thresholds (audiogram values in Fig. 1) over the frequency range covered by the harmonics. For example, the DLCs for the complex tone with harmonics 1-12 with $f_{0}=50 \mathrm{~Hz}$ were positively correlated with the absolute thresholds up to $1 \mathrm{kHz}$ ( $r=0.59,0.44$, and 0.40 at 250,500 , and $1000 \mathrm{~Hz}$, respectively), but showed essentially no correlation for frequencies above $1 \mathrm{kHz}$. For $f_{0}=400 \mathrm{~Hz}$, the correlations were about 0.5 for frequencies from 250 to 2000 $\mathrm{Hz}$, but were close to 0 at 4 and $8 \mathrm{kHz}$. These correlations probably reflect the fact that the stimuli were presented at a fixed SPL, so sensation levels were lower for subjects with higher absolute thresholds.

We turn now to a consideration of the effects on DLCs of the relative phases of the components. For all four subject groups, DLCs were, on average, larger for components added in alternating phase than for components added in cosine phase. As described above, this phase effect was significant for each subject group. The mean DLCs for each group, harmonic complex and phase are shown in Fig. 8; results have been averaged across $f_{0}$ 's, since only one group showed 


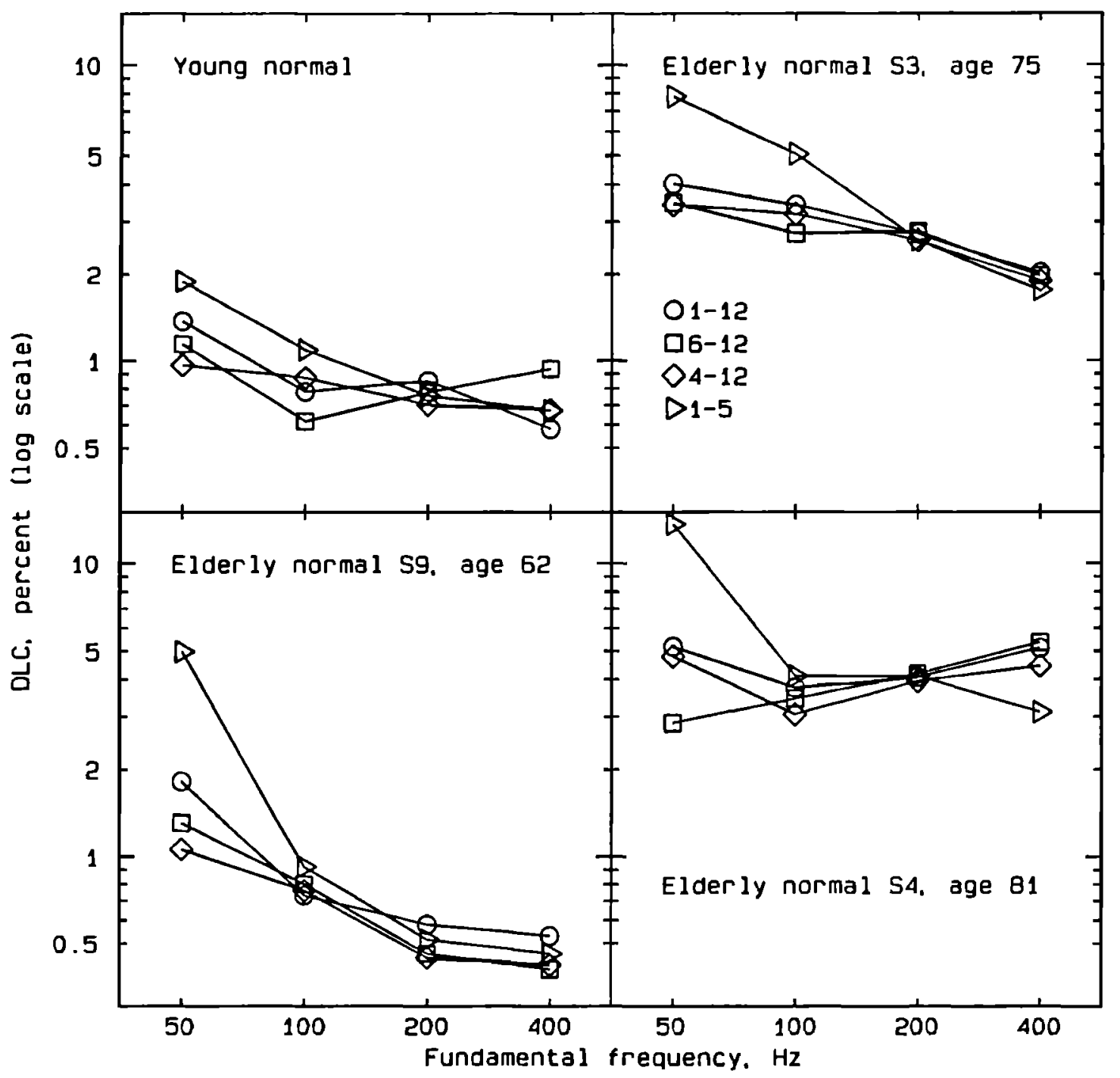

FIG. 7. As in Fig. 4, but showing the results for the young normal group and for three individual subjects from the elderly normal group, chosen because their auditory filters were almost normal at low center frequencies.

a significant interaction of phase with $f_{0}$.

In every case shown, DLCs are larger for alternating phase than for cosine phase, but the effects overall are rather small. This is somewhat misleading, however, in indicating the influence of phase, since the direction of the effect (whether the change from cosine to alternating phase made performance worse or better) varied in an idiosyncratic way across subjects, $f_{0} s$, and harmonic contents. Phase effects for individual subjects were often considerably larger than indicated in Fig. 8.

\section{Relationship between the DLFs and the DLCs}

The DLCs at all $f_{0}$ s were positively correlated with the DLFs at all frequencies tested. This probably partly reflects a general ability to perform pitch discrimination tasks, an ability that varies from subject to subject. However, it is of interest that the DLCs at a given $f_{0}$ were more highly correlated with the DLFs at frequencies corresponding to higher

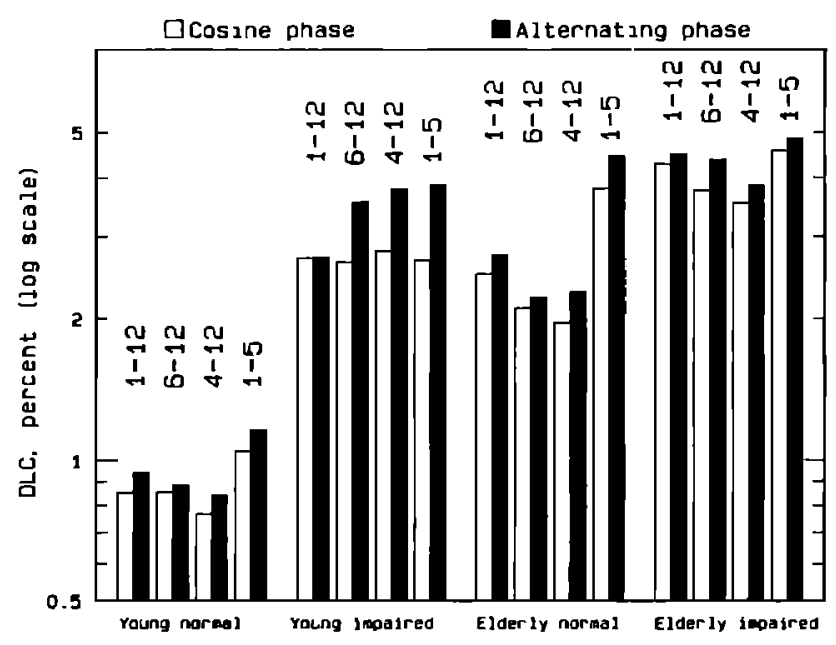

FIG. 8. DLCs averaged across $f_{0}$ 's, for complex tones with components added in cosine phase (open bars) and alternating phase (solid bars). Results are shown separately for each group of subjects. 
harmonics than with DLFs corresponding to $f_{0}$. For example, considering the results of the two hearing-impaired groups together, the DLCs at $f_{0}=100 \mathrm{~Hz}$ were highly correlated with the DLFs at 200,400 , and $800 \mathrm{~Hz}(r=0.83$, 0.82 , and 0.90 , respectively ), but were only weakly correlated with the DLF at $100 \mathrm{~Hz}(r=0.26)$. This is consistent with the evidence presented earlier that the pitch discrimination of these complex tones depended strongly on the processing of information from higher harmonics.

\section{GENERAL DISCUSSION}

These results confirm earlier results in showing that pitch discrimination of both pure and complex tones is worse than normal in subjects with sensorineural hearing loss. Our results do not show any clear differences between the young and elderly hearing-impaired subjects. However, some of the elderly subjects with near-normal absolute thresholds did show markedly impaired pitch discrimination of both pure and complex tones. This poor discrimination could reflect a general cognitive difficulty rather than a defect in the basic processing of spectral or temporal information. However, our three-interval, three-alternative forced-choice task was deliberately chosen to minimize this possibility. Subjects did not have to identify the direction of a pitch change; all they had to do was to pick the "odd" interval. Furthermore, it is clear that subjects were able to master the three-interval task, since they all gave consistent and reproducible results in the measurement of masked and absolute thresholds. Thus it seems more likely that the poor performance of some of the elderly normal subjects reflects a difficulty specific to pitch, possibly because of a reduced ability to process temporal information.

The results for pure-tone frequency discrimination indicate a partial dissociation of frequency discrimination and frequency selectivity, at least at low frequencies. Some subjects with near-normal auditory filters had large DLFs, while another with broad auditory filters had near-normal DLFs. This is inconsistent with a pure place theory of frequency discrimination, but is consistent with the idea that frequency discrimination at low frequencies depends at least partly on temporal processing.

Several aspects of the results for complex tones support spectrotemporal theories of pitch perception as opposed to purely spectral theories. First, at low $f_{0}$ 's, discrimination was better for complexes containing high harmonics than for complexes containing only low harmonics. This suggests that higher, unresolved harmonics are dominant in determining pitch at low $f_{0}$ 's for both normal and hearing-impaired subjects. Second, DLCs for the elderly impaired and elderly normal subjects were sometimes larger for the complex with harmonics 1-12 than for the complexes with harmonics 6-12 or 4-12. Thus adding lower harmonics can impair performance. Results similar to this have been reported by Moore and Glasberg $(1988,1990 a)$. This is difficult to explain in terms of spectral theories. It can be explained by spectrotemporal theories on the assumption that, for subjects with broad auditory filters, adding low harmonics may produce more complex waveforms at the outputs of auditory filters responding to higher harmonics. This may make sub- sequent temporal analysis more difficult.

A third aspect of the results supporting spectrotemporal theories is the finding of significant effects of the relative phases of the components. The effects were present for all groups, but were somewhat larger for the impaired groups. Some workers (e.g., Buunen et al., 1974) have suggested that the effects of relative phase on pitch can be explained in terms of combination tones, particularly the cubic difference tone, $2 f_{1}-f_{2}$. Changing the phases of the components in a complex tone may affect the relative levels of the combination tones, which in turn might affect pitch. However, this explanation seems unlikely to apply to our results, since the levels of combination tones are lower in hearing impaired than in normal subjects (Leshowitz and Lindstrom, 1977), but the phase effects are generally larger for impaired than for normal subjects. The effects of phase on the DLCs most likely reflect a sensitivity to the time structure of the waveforms at the outputs of the auditory filters, as proposed by spectrotemporal theories of pitch.

In conclusion, the frequency discrimination of both pure and complex tones is worse than normal in young and elderly hearing-impaired subjects. In addition, some elderly subjects with normal absolute thresholds and normal auditory filters show impaired frequency discrimination. This may reduce the ability to take advantage of prosodic cues during audiovisual speech perception. The results for both pure and complex tones are more consistent with spectrotemporal theories of pitch perception than with spectral theories of pitch perception.

\section{ACKNOWLEDGMENTS}

This work was supported in part by grants from The AARP Andrus Foundation, The Deafness Research Foundation, UNC-CH Medical School Foundation, UNC Faculty Research Council, The Burroughs Wellcome Fund, and the Medical Research Council (U.K.). We thank Harold C. Pillsbury for otological examinations and consultation with respect to medical aspects of the study; Joseph Hall and John Grose for help in generating the noise stimuli and for advice on programming and instrumentation; Susan Mahanes, Elizabeth Poth, Kathleen Berry, Melissa Page, Margaret Casteen and Martha Sloan-Clontz for assistance in data collection; Tom Baer, Brian Glasberg, Roy Patterson, Michael Shailer, and Michael Stone for helpful comments on an earlier version of this paper; and Brian Glasberg for assistance with data analysis and preparation of figures. We also thank our subjects, including those from the Carol Woods Retirement Community, for their many hours of service. Finally, we thank an anonymous reviewer and a reviewer whose signature is probably that of Ed Burns for their constructive criticism.

Alvey, N., Galway, N., and Lane, P. (1982). An Introduction to GENSTAT (Academic, London).

Buunen, T. J. F., Festen, J. M., Bilsen, F. A., and Brink, G. van den (1974). "Phase effects in a three-component signal," J. Acoust. Soc. Am. 55, 297-303.

Glasberg, B. R., and Moore, B. C. J. (1986). "Auditory filter shapes in subjects with unilateral and bilateral cochlear impairments," J. Acoust. Soc. Am. 79, 1020-1033.

Glasberg. B. R., and Moore, B. C. J. (1989). "Psychoacoustic abilities of 
subjects with unilateral and bilateral cochlear hearing impairments and their relationship to the ability to understand speech," Scand. Audiol. Suppl. 32, 1-25.

Goldstein, J. L. (1973). “An optimum processor theory for the central formation of the pitch of complex tones," J. Acoust. Soc. Am. 54, 14961516.

Goldstein, J. L., and Srulovicz, P. (1977). “Auditory-nerve spike intervals as an adequate basis for aural frequency measurement," in Psychophysics and Physiology of Hearing, edited by E. F. Evans and J. P. Wilson (Academic, London).

Grant, K. W., Ardell, L. H., Kuhl, P. K., and Sparks, D. W. (1985). "The contribution of fundamental frequency, amplitude envelope, and voicing duration cues to speechreading in normal-hearing subjects," J. Acoust. Soc. Am. 77, 671-677.

Hoekstra, A. and Ritsma, R. J. (1977). "Perceptive hearing loss and frequency selectivity," in Psychophysics and Physiology of Hearing, edited by E. F. Evans and J. P. Wilson (Academic, London).

Horst, J. W. (1987). "Frequency discrimination of complex signals, frequency selectivity and speech perception in hearing-impaired subjects," J. Acoust. Soc. Am. 82, 874-885.

Houtsma, A. J. M., and Smurzynski, J. (1990). "Pitch identification and discrimination for complex tones with many harmonics," J. Acoust. Soc. Am. 87, 304-310.

Humes, L. E., and Christopherson, L. (1991). "Speech identification difficulties of hearing-impaired elderly persons: The contributions of auditory processing deficits," J. Speech Hear. Res. 34, 686-693.

Kendall, M. G., and Stuart, A. (1967). The Advanced Theory of Statistics Vol. II (Griffin, London), 2nd ed.

Leshowitz, B. H., and Lindstrom, R. ( 1977). "Measurement of nonlinearities in listeners with sensorineural hearing loss," in Psychophysics and Physiology of Hearing, edited by E. F. Evans and J. P. Wilson ( Academic, London).

Meddis, R., and Hewitt, M. (1991). "Virtual pitch and phase sensitivity studied using a computer model of the auditory periphery: Pitch identification," J. Acoust. Soc. Am. 89, 2866-2882.

Moore, B. C. J. (1973). "Frequency difference limens for short-duration tones," J. Acoust. Soc. Am. 54, 610-619.

Moore, B. C. J. (1977). "Effects of relative phase of the components on the pitch of three-component complex tones," in Psychophysics and Physiolo$g y$ of Hearing, edited by E. F. Evans and J. P. Wilson (Academic, London).

Moore, B. C. J. (1982). An Introduction to the Psychology of Hearing (Academic, London), 2nd ed.

Moore, B. C. J. (1989). An Introduction to the Psychology of Hearing (Academic, London ), 3rd ed.

Moore, B. C. J., and Glasberg, B. R. (1986a). "The role of frequency selectivity in the perception of loudness, pitch and time," in Frequency Selectivity in Hearing, edited by B. C. J. Moore (Academic, London).

Moore, B. C. J., and Glasberg, B. R. (1986b). "The relationship between frequency selectivity and frequency discrimination for subjects with unilateral and bilateral cochlear impairments," in Auditory Frequency Selectivity, edited by B. C. J. Moore and R. D. Patterson (Plenum, New York).

Moore, B. C. J., and Glasberg, B. R. (1987). "Pitch perception and phase sensitivity for subjects with unilateral and bilateral cochlear hearing impairments," in Clinical Audiology '87, edited by A. Quaranta (Laterza, Bari, Italy).

Moore, B. C. J., and Glasberg, B. R. (1988). "Effects of the relative phase of the components on the pitch discrimination of complex tones by subjects with unilateral cochlear impairments," in Basic Issues in Hearing, edited by H. Duifhuis, J. W. Horst, and H. P. Wit (Academic, London).

Moore, B. C. J. and Glasberg, B. R. (1990a). "Frequency selectivity in subjects with cochlear hearing loss and its effects on pitch discrimination and phase sensitivity," in Advances in Audiologv, Vol. 7. Cochlear Mechanisms and Otoacoustic Emissions, edited by F. Grandori, G. Cianfrone, and D. T. Kemp (Karger, Basel, Switzerland).

Moore, B. C. J., and Glasberg, B. R. (1990b). "Frequency discrimination of complex tones with overlapping and non-overlapping harmonics," $\mathbf{J}$. Acoust. Soc. Am. 87, 2163-2177.

Moore, B. C. J., Glasberg, B. R., and Shailer, M. J. (1984). "Frequency and intensity difference limens for harmonies within complex tones," $J$. Acoust. Soc. Am. 75, 550-561.

Moore, B. C. J., Glasberg, B. R., and Peters, R. W. (1985). "Relative dominance of individual partials in determining the pitch of complex tones," $\mathrm{J}$.
Acoust. Soc. Am. 77, 1853-1860.

Moore, B. C. J., Peters, R. W., and Glasberg, B. R. (1990). "Auditory filter shapes at low center frequencies," J. Acoust. Soc. Am. 88, 132-140.

Moore, B. C. J., and Rosen, S. M. (1979). "Tune recognition with reduced pitch and interval information," Q. J. Exp. Psychol. 31, 229-240.

Nelson, D. A., Stanton, M. E., and Freyman, R. L. (1983). "A general equation describing frequency discrimination as a function of frequency and sensation level," J. Acoust. Soc. Am. 73, 2117-2123.

Noorden, L. P. A. S. van (1982). "Two channel pitch perception," in Music, Mind and Brain, edited by M. Clynes (Plenum, New York).

Patterson, R. D. (1976). “Auditory filter shapes derived with noise stimuli," J. Acoust. Soc. Am. 59, 640-654.

Patterson, R. D. (1987). "A pulse ribbon model of peripheral auditory processing," in Auditory Processing of Complex Sounds, edited by W. A. Yost and C. S. Watson (Erlbaum, Hillsdale, NJ).

Patterson, R. D., and Moore, B. C. J. (1986). "Auditory filters and excitation patterns as representations of frequency resolution," in Frequency Selectivity in Hearing, edited by B. C. J. Moore (Academic, London).

Patterson, R. D., Nimmo-Smith, I., Weber, D. L., and Milroy, R. (1982). "The deterioration of hearing with age: Frequency selectivity, the critıcal ratio, the audiogram, and speech threshold," J. Acoust. Soc. Am. 72, 1788-1803.

Patterson, R. D., and Wightman, F. L. (1976). "Residue pitch as a function of component spacing," J. Acoust. Soc. Am. 59, 1450-1459.

Peters, R. W., and Moore, B. C. J. (1992a). "Auditory filter shapes at low center frequencies in young and elderly hearing-impaired subjects," $J$. Acoust. Soc. Am. 91, 256-266.

Peters, R. W., and Moore, B. C. J. (1991b). "Auditory filters and aging: filters when audiometric thresholds are normal," In 9th International Symposium on Hearing: A uditory Physiology and Perception, edited by Y. Cazals, L. Demany, and K. Horner (Pergamon, Oxford) (in press).

Pick, G. F., Evans E. F., and Wilson, J. P. (1977). "Frequency resolution in patients with hearing loss of cochlear origin," in Psychophysics and Physiology of Hearing, edited by E. F. Evans and J. P. Wilson (Academic, London).

Plomp, R. (1964). "The ear as a frequency analyzer," J. Acoust. Soc. Am. $36,1628-1636$.

Plomp, R. (1967). "Pitch of complex tones," J. Acoust. Soc. Am. 41, 15261533.

Risberg. A. (1974). "The importance of prosodic spuech elements for the lipreader," in Visual and Audio-Visual Perception of Speech, edited by $\mathrm{H}$. B. Nielsen and E. Kampp, Scand. Audiol. Suppl. 4 (Almquist and Wiksell, Stockholm)

Ritsma, R. J. (1962). "Existence region of the tonal residue. I," J. Acoust. Soc. Am. 34, 237-238.

Ritsma, R. J. (1963). "Existence region of the tonal residue. II," J. Acoust. Soc. Am. 35, 124l-1245.

Ritsma, R. J. (1967). "Frequencies dominant in the perception of the pttch of complex sounds," J. Acoust. Soc. Am. 42, 191-198.

Rosen, S., and Fourcin, A. (1986). "Frequency selectivity and the perception of speech," in Frequency Selectivity in Hearing, edited by B. C. J. Moore (Academic, London).

Rosen, S., Fourcin, A. J., and Moore, B. C. J. (1981). "Voice pitch as an aid to lip-reading," Nature, London, 291, 150-152.

Srulovicz, P., and Goldstein, J. L. (1983). "A central spectrum model: A synthesis of auditory-nerve timing and place cues in monaural communication of frequency spectrum," J. Acoust. Soc. Am. 73, 1266-1276.

Terhardt, E. (1974). "Pitch, consonance and harmony," J. Acoust. Soc. Am. 55, 1061-1069.

Terhardt, E. (1977). "Evaluation of the susceptibility of virtual pitch to phase," in Psychophysics and Physiology of Hearing, edited by E. F. Evans and J. P. Wilson (Academic, London), pp. 359-360.

Tyler, R. S. (1986). "Frequency resolution in hearing-impaired listeners," in Frequency Selectivity in Hearing, edited by B. C. J. Moore (Academic, London).

Tyler, R. S., Wood, E. J., and Fernandes, M. A. (1983). "Frequency resolution and discrimination of constant and dynamic tones in normal and hearing-impaired listeners," J. Acoust. Soc. Am. 74, 1190-1199.

Wier, C. C., Jesteadt, W., and Green, D. M. (1977). "Frequency discrimination as a function of frequency and sensation level," J. Acoust. Soc. Am. 61, 178-184.

Zurek, P. M., and Formby, C. (1981). "Frequency discrimination ability of hearing-impaired listeners," J. Speech. Hear. Res. 46, 108-112 